\title{
Coronary Collaterals Predict Improved Survival and Allograft Function in Patients with Coronary Allograft Vasculopathy
}

\author{
Kory J. Lavine, MD, PhD ${ }^{1,3}$, Marc Sintek, MD ${ }^{1}$, Eric Novak, MS ${ }^{1}$, Gregory Ewald, MD ${ }^{1}$, \\ Edward Geltman, MD1 , Susan Joseph, MD ${ }^{1}$, John Pfeifer, MD $^{2}$, and Douglas Mann, MD ${ }^{1}$ \\ ${ }^{1}$ Division of Cardiology, Barnes Jewish Hospital, Washington University School of Medicine, \\ Campus Box 8086, 660 South Euclid, St Louis, MO 63112 \\ ${ }^{2}$ Department of Pathology, Barnes Jewish Hospital, Washington University School of Medicine, \\ Campus Box 8086, 660 South Euclid, St Louis, MO 63112
}

\begin{abstract}
Background-Despite improvements in the care of patients who have received cardiac transplants, coronary allograft vasculopathy (CAV) remains the most prevalent cause of late allograft failure and cardiac mortality. Few proven therapies are available for this important disease. The presence of coronary collaterals imparts a favorable prognosis in patients with native ischemic heart disease; however, the impact of collaterals in CAV is unknown.
\end{abstract}

Methods and results-To determine whether the development of coronary collaterals is associated with improved outcomes in patients with $\mathrm{CAV}$, we performed a retrospective analysis of patients followed in the heart transplant program at Barnes Jewish Hospital from 1994-2008. The primary endpoints included all cause mortality and the composite of all cause mortality, retransplantation, and inotrope dependence. We screened 493 patients and identified 59 (12\%) subjects with moderate to severe CAV. Angiographically visible coronary collaterals were present in 34 (57\%) subjects. Kaplan-Meier and Cox multivariable analyses revealed that patients with collaterals had reduced incidence of all cause mortality HR $0.20, p<0.001$ and the composite endpoint HR 0.17, $<<0.001$. In addition, patients with collaterals had less severe heart failure symptoms as measured by NYHA class. Immunostaining of biopsy specimens revealed that among patients with CAV, the presence of coronary collaterals correlated with increased microvascular density, reduced fibrosis and lower LVEDP.

Conclusions-Together, these data demonstrate that the presence of coronary collaterals predicts a favorable prognosis in patients with $\mathrm{CAV}$ and suggests that interventions aimed at promoting collateral and microvascular growth may serve as effective therapies for this disease.

\section{Keywords}

collateral; transplant; vasculopathy; transplant; coronary

\section{Introduction}

Recent advances in immunosuppression have significantly decreased the incidence of death and allograft failure early following transplantation ${ }^{1}$. However, the prevalence of late allograft failure and associated mortality have remained unchanged over the past 10 years ${ }^{1}$. The major causes of late allograft failure include, coronary allograft vasculopathy (CAV),

\footnotetext{
${ }^{3}$ Corresponding author, klavine@ dom.wustl.edu, Phone: 314 362-8901 Fax: 314 362-0186.

Disclosures. none
} 
malignancies, and nonspecific graft failure ${ }^{2-4}$. As the opportunities for retransplantation continue to decline, the development of novel therapies aimed at preserving allograft function has become an important and unmet clinical need.

Among the causes of late allograft failure, CAV continues to be a challenge from both a diagnostic and treatment perspective. CAV is typically diagnosed either on routine screening angiograms or in the setting of heart failure. Coronary angiography is the gold standard for diagnosis where characteristic pruning of the distal coronary arteries is the predominate feature. Additionally, lesions in more proximal coronary arteries may be present ${ }^{5-7}$. Intravascular ultrasound has increasingly been used for earlier diagnosis and monitoring of disease progression ${ }^{8-10}$. Unlike traditional coronary artery disease, CAV tends to diffusely affect the distal coronary vasculature ${ }^{6}$.

To date there are few effective treatments available for patients with CAV. Of these, statin therapy appears to be the most efficacious and appears to reduce disease progression and mortality in randomized studies ${ }^{12,13}$. Other prospective trials have demonstrated smaller improvement in outcomes for patients treated with sirolimus, everolimus, mycophenolate mofetil, ganciclovir, calcium channel blockers and angiotensin converting enzyme inhibitors ${ }^{14-19}$. Coronary revascularization is limited to percutaneous intervention and serves as a palliative treatment modality in these patients ${ }^{20-22}$. Despite use of these therapies, CAV remains an important cause of allograft failure and the only definitive therapy is retransplantation.

Previous studies have demonstrated that the development of coronary collateral blood vessels portends a favorable prognosis in patients with coronary artery disease and ischemic cardiomyopathy ${ }^{23-27}$. The prognostic impact of coronary collateral development has not yet been studied in patients with CAV. We hypothesized that the development of coronary collateral vasculature is similarly associated with favorable outcomes in patients with CAV.

To test this hypothesis, we performed a retrospective cohort analysis of all patients who were cared for in the heart transplant program at Barnes Jewish Hospital between 1994 and 2008. Here, we show that patients with CAV who develop coronary collateral vasculature have significantly improved outcomes compared to those without collaterals. In addition, we reveal that patients with CAV who develop collaterals have increased microvascular density, reduced myocardial fibrosis and decreased LVEDP.

\section{Materials and Methods}

\section{Study design}

To identify patients with significant CAV, we performed a retrospective analysis of all patients who either received a cardiac transplant at Barnes Jewish Hospital between 1994 and 2008 or received a heart transplant elsewhere and were followed in our post-transplant program between 1994 and 2008. Patients with significant CAV included those with moderate to severe transplant vasculopathy as defined by the ISHLT ${ }^{28}$. This distinction was made in order to exclude patients with mild or trivial disease. Prior to study initiation, institutional IRB approval was obtained and the study conducted in accordance to the approved protocol. The electronic medical record was queried to identify patients with moderate or severe coronary allograft vasculopathy. Available angiograms were reviewed by committee to confirm the presence of moderate or severe CAV as defined by the ISHLT.

Patients were then divided into two groups: 1) patients with coronary collaterals and 2) patients without coronary collaterals. The presence of coronary collaterals was determined 
by reviewing the coronary angiogram obtained at the time of CAV diagnosis. Angiograms were reviewed by committee and patients allocated into the above groups.

Patients were included in the study if they had moderate or severe CAV, 3 years of clinical follow up (unless a clinical endpoint was reached), and available coronary angiograms. Exclusion criteria included inadequate follow up, angiographic, or clinical data. Standard demographic information was obtained from the medical record including age, sex, reason for transplantation, cardiovascular comorbidities, transplant and heart failure medicines, rejection episodes, CMV status, blood type, echocardiographic data, stress testing, LV end diastolic pressure, heart rate and blood pressure as derived from diagnostic catheterization data. Data describing donor characteristics and HLA matching were not available for all patients included in this study and was unable to be rigorously examined.

\section{Endpoints}

We utilized two primary endpoints; all cause mortality and the composite of all cause mortality, chronic inotrope therapy, and need for retransplantation. Each of these endpoints was pre-specified in the study protocol. Mortality events were identified through clinical data and confirmed using the social security death index. Chronic inotrope therapy and retransplantation were defined using available clinical data.

Secondary endpoints included two independent measures of heart failure severity. New York Heart Association (NYHA) class, as documented in the clinical record at the time of CAV diagnosis, served as a surrogate for functional class and exertional capacity. LV end diastolic pressure (LVEDP) recorded at the time of coronary angiography and CAV diagnosis was used as an objective assessment of allograft dysfunction. Each of these secondary endpoints was pre-specified in the study protocol.

\section{Statistical analysis}

Statistical analysis was performed with SAS v9.3 (SAS Institute, Cary, NC). Fisher's exact test was used to identify differences in demographic categorical variables between patients with and without collaterals. Wilcoxon-Mann-Whitney tests were used for continuous variables. Kaplan-Meier curves were generated for all cause mortality and the composite outcome. Transplantation date was considered to be time zero. Patient groups (with and without collaterals) were compared via the log rank test. The Cochran-Armitage trend test was used to examine the percent of collaterals by NYHA class.

Cox proportional hazards multivariable models were created for each of the co-primary outcomes to adjust for covariates and potential confounding variables. Schoenfeld residuals were used to check the proportionality assumption for all independent variables. The hazard ratio (HR) and 95\% confidence intervals were reported from these models. The interaction between time from transplant to diagnosis and time was included to adjust for nonproportionality.

Student's t-test and ANOVA was used to compare antibody immunostaining results between different patient groups. The coefficient of determination $\left(\mathrm{r}^{2}\right)$ was used to describe the relationship between LVEDP, picrosirius red area stained, and blood vessel area stained.

\section{Biopsy specimens}

Myocardial biopsy specimens that were previously collected for routine clinical care were obtained from the department of pathology. Collected tissues were previously fixed in $10 \%$ formalin and stored in paraffin blocks. For patients with CAV, specimens were collected within 1-2 months of diagnosis and demonstrated minimal acute cellular rejection (grade $\mathrm{O}$ 
or $1 \mathrm{~A}$ rejection). A control group was created by randomly selecting post-transplant patients without CAV matched only by year of cardiac transplantation. For each patient, the most recent biopsy specimen was obtained. Patients were excluded if they experienced recurrent rejection, defined as no more than 2 episodes of $2 R$ or $3 R$ rejection and no episodes of AMR. Demographic and outcome data were collected for the control group and compared to patients with CAV (Supplemental Table 1).

\section{Histology and immunohistochemstrsy}

Myocardial biopsy specimens were cut into 4 micron sections using standard techniques and mounted on glass slides. Each paraffin block typically contained 3-4 myocardial specimens. Immunohistochemistry was performed using the following antibodies CD31 (1A10), CD34 (QBEnd/10), and SMA (1A4). The primary antibody was detected using HRP conjugated secondary antibodies and DAB substrate. SMA immunostained sections were counterstained with hematoxylin. Picrosirius red staining was performed using standard techniques. These assays were performed by the Anatomic and Molecular Pathology core as a component of the Institute of Clinical and Translational Science (ICTS) at Washington University School of Medicine.

To identify the microvasculature, our initial experiments utilized CD31 (PECAM) and CD34 antibodies as well as UEA I Lectin. Each of these reagents specifically labeled endothelial cells, however, CD34 immunostaining appeared to give the most consistent and robust signal particularly in older specimens. For this reason we performed the majority of the analyses using the CD34 antibody. CD31 immunostaining and UEA1 Lectin staining demonstrated consistent findings (data not shown). Smooth muscle actin (SMA) immunostaining was used to identify arterioles.

For each antibody, 2-3 sections were stained. Positive staining was quantified with an automated software package as the percent area stained. Manual quantification of microvascular density demonstrated a robust correlation with the automated method (data not shown). The following protocol was utilized. For each myocardial specimen, 2 independent fields from each of the stained sections were photographed at 20X magnification. Image $\mathbf{J}$ software was used to quantify the percent area stained. The resulting measurements (typically 6-8) were then averaged to produce a single value to be used in the final analysis. Pircosirius red staining was quantified using an identical method. SMA immunostaining was quantified by manually counting the number of SMA containing blood vessels per 20X field. All specimens were stained, photographed and quantified in blinded fashion.

\section{Results}

\section{Study design and baseline characteristics}

In total, we identified 485 patients who were transplanted between 1994 and 2008, cared for in our post-transplant program, and had at least 3 years of clinical follow up. A chart review identified 63 patients who had moderate or severe CAV. Angiographic data were available for 59 of these patients. Coronary angiograms obtained at the time of CAV diagnosis were then reviewed by committee to verify the presence of moderate or severe CAV. Of the 59 angiograms available for review, all were determined to meet criteria for moderate or severe CAV. Patients were divided into those with and without angiographically visible collaterals. Examples of a normal coronary angiogram compared to angiograms demonstrating moderate to severe CAV with and without collateral formation are shown in Figure 1A-C. Of the 59 patients with significant $\mathrm{CAV}, 34$ individuals had angiographically visible collateral blood vessels and 25 individuals had no collaterals (Figure 1D). 
Examination of baseline demographics demonstrated no significant differences between CAV patients with and without collaterals for several known risk factors including age, race, diabetes, CMV status, rejection events, heart failure and immunosuppressive medications. However, there was a significant difference seen between these two groups with respect to timing of diagnosis of CAV (Table 1). Patients with CAV but without coronary collateral blood vessels were diagnosed considerably earlier than those with collateral vasculature, 4.8 versus 7.9 years, respectively $(\mathrm{p}<.001)$. In addition, patients without collaterals had increased heart rate, increased diastolic blood pressure, and reduced pulse pressure compared to patients without collaterals.

\section{Improved outcomes in CAV patients with coronary collateral vasculature}

To determine whether development of coronary collateral blood vessels is associated with improved outcomes in patients with CAV, we performed a time to event analysis using two pre-specified primary outcomes; all cause mortality and the composite of all cause mortality, retransplantation, and chronic inotrope use. For these analyses the time of transplantation was considered to be time zero. Kaplan-Meier analysis revealed significantly reduced rates of both all cause mortality and the composite endpoint in patients who develop coronary collaterals, both $p<0.001$ (Figure 2). The composite endpoint was primarily driven by all cause mortality. Death occurred in 10 patients (29\%) in the collateral group and 20 patients $(80 \%)$ in the no collateral group ( $\mathrm{p}<.001)$. With respect to retransplantation, 1 patient $(2.9 \%)$ underwent a second transplant in the collateral group, while 2 patients $(8.0 \%)$ required a second transplant in the no collateral group. Chronic inotropic therapy was initiated in 1 patient from each of the collateral and no collateral groups ( $2.9 \%$ and $4.2 \%, \mathrm{p}=\mathrm{NS}$ ).

Univariate analysis demonstrated that coronary collaterals, statin therapy, male sex, and higher pulse pressure were associated with improved outcomes in patients with CAV. In contrast, worse NYHA class, higher LVEDP, lower ejection fraction, higher heart rate, rejection, prednisone, and azathioprine therapy were associated with worse outcomes in patients with CAV (Table 2). Among the variables examined, NYHA class and the presence of coronary collaterals represented the two most robust predictors of outcomes.

Cox multivariable models revealed that the presence of coronary collaterals was independently associated with improved outcomes in patients with CAV. We utilized two separate analyses to test whether coronary collateral development was an independent predictor of the primary endpoints. Within our cohort, 35 events contributed to the composite endpoint and 30 events contributed to all cause mortality. Based on prior studies demonstrating that multivariable models are most accurate when they include 10 events per variable $^{29-31}$, we first constructed a multivariable model that included the three variables that most robustly predicted outcomes. This analysis demonstrated that angiographically visible collaterals were associated with all cause mortality (HR 0.20, 95\% CI 0.08-0.54, $\mathrm{p}=0.001$ ) and the composite endpoint (HR 0.29, 95\% CI 0.11-0.74, $\mathrm{p}=0.010)$ independent of NYHA class and prednisone therapy (Table 3). To confirm these findings, we developed a series of multivariable models which tested whether the presence of collaterals was independently associated with outcomes after adjusting for each individual variable. This analysis revealed that the presence of coronary collaterals was associated with a lower incidence of all cause mortality and the composite endpoint independent of NYHA class, ejection fraction, LVEDP, heart rate, pulse pressure, rejection, male sex, statin, azathioprine, and prednisone therapy (Table 3).

\section{Reduced heart failure symptoms in patients with coronary collaterals}

To determine whether the presence of coronary collateral blood vessels impacts on heart failure symptoms in patients with CAV, we tested for an association between New York 
Heart Association (NYHA) class, as documented in the clinical record at the time of CAV diagnosis, and the development of coronary collaterals. Consistent with improved outcomes in CAV patients with collaterals, the presence of coronary collaterals was associated with reduced NYHA class $(\mathrm{p}<0.001$, Figure $3 \mathrm{~A})$. The majority of CAV patients with coronary collaterals had either NYHA class I or II symptoms (29\% and 56\%, respectively). A small minority of patients with collaterals reported NYHA class III or IV symptoms (12\% and 3\%, respectively). In contrast, CAV patients without collaterals had significantly fewer patients with NYHA class I or II symptoms (4\% and $27 \%$, respectively). The majority of CAV patients without collaterals had NYHA class III or IV symptoms $(58 \%$ and $11 \%$, respectively). Trend analysis confirmed that the development of coronary collaterals in patients with CAV was associated with reduced NYHA class severity (Figure 3B).

\section{Late diagnosis is associated with coronary collateral development}

As mentioned previously, patients with CAV who form coronary collateral vasculature tended to be diagnosed later than those without collaterals ( $4.8 \pm 3.1$ versus $7.9 \pm 3.6$ years following transplant, $\mathrm{p}=0.001$ ) (Figure 4A). Of note, patients in this study underwent coronary angiography based on a strategy driven by a combination of routine screening, high risk stress testing, or in the context of progressive heart failure symptoms. Thus, collateral formation may protect patients from the development of symptoms and lead to a later timing of diagnosis. Alternatively, an earlier diagnosis of CAV may indicate more aggressive disease. In this setting, there may not be adequate time to form collateral vasculature.

To determine whether collateral formation was predictive of outcomes independent of the timing of diagnosis, we performed two additional analyses. Kaplan-Meier analysis using the time of CAV diagnosis as time zero demonstrated that patients with collaterals have a reduced incidence of all cause mortality as well as the composite end point compared to those without collaterals (Figure 4B). Furthermore, Cox multivariable models demonstrated that coronary collateral development and the time to diagnosis were independently associated with improved outcomes supporting the notion that coronary collaterals are protective and may delay the diagnosis of CAV (Figure 4C). A longer time period between treatment and diagnosis was associated with improved outcomes, but this association weakened over time.

\section{Microvascular density}

To gain further insight into why CAV patients with coronary collaterals have improved outcomes compared to those without collaterals, we obtained RV endomyocardial biopsy specimens from patients included in this study. Selected biopsy specimens were collected within 1-2 months from the time that CAV was diagnosed and demonstrated minimal acute cellular rejection (grade $\mathrm{O}$ or $1 \mathrm{~A}$ rejection). Adequate endomyocardial specimens were available from 22/25 (88\%) patients in the no collateral group and from 31/34 (91\%) patients in the collateral group. We also included 23 control samples that were matched based on the timing of transplantation. Demographic and outcome data comparing control and CAV patients are provided in Supplemental Table 1. Control patients tended to be older than those with CAV and had a lower incidence of diabetes, a well known risk factor for $\mathrm{CAV}$. The groups were otherwise well balanced with respect to demographic data. As expected, patients with CAV tended to have a higher incidence of prior $\mathrm{PCI}$ and heart failure as demonstrated by decreased systolic BP, higher heart rate, higher LVEDP, worse NYHA class, and systolic dysfunction.

To examine alterations in microvascular density, we performed immunohistochemical staining with antibodies that specifically recognize endothelial cells and smooth muscle cell 
actin. Immunostaining for CD34 revealed that compared to controls, patients with CAV had significantly reduced microvasculature (Figure $5 \mathrm{~A}-\mathrm{C}, \mathrm{G}$ ). The largest difference in microvascular density occurred between control subjects and CAV patients without collaterals where there was a greater than 2 fold difference between groups $(7.1 \pm 1.1 \%$ versus $3.2 \pm 0.9 \%, \mathrm{p}<0.001)$. While CAV patients with collaterals had reduced microvascular density compared to control subjects $(5.2 \pm 1.4 \%$ versus $7.1 \pm 1.1 \%$, p $<0.001)$, these patients had significantly increased microvascular density compared to CAV patients without collaterals $(5.2 \pm 1.4 \%$ versus $3.2 \pm 0.9 \%, \mathrm{p}=0.001)$. Together these data reveal that microvascular density is significantly reduced in patients with CAV and suggest that there is a significant association between collateral development and preservation of microvascular density.

Smooth muscle actin immunostaining demonstrated that compared to controls, patients with CAV had significantly reduced arteriolar density (Figure 5D-F,H). Arteriolar density was quantified by counting the number of SMA+ blood vessels per 20X field. CAV patients without collaterals had a statistically significant reduction in arteriolar density compared to control subjects $(5.8 \pm 1.4$ versus $7.8 \pm 1.4 \mathrm{SMA}+$ vessels/20X field, $\mathrm{p}<0.001)$. In contrast, there was no statistical difference in arteriolar density between CAV patients with collaterals and control subjects $(7.0 \pm 1.9$ versus $7.8 \pm 1.4 \mathrm{SMA}+$ vessels/20X field, $\mathrm{p}=0.10)$.

\section{Myocardial fibrosis}

Myocardial fibrosis is predictive of poor outcomes in patients who have been subjected to various cardiac insults ${ }^{32,33}$. To characterize changes in myocardial fibrosis, we performed Picrosirius red staining and quantified the percent area stained. Compared to control subjects, patients with CAV demonstrated evidence of increased interstitial fibrosis (Figure $6 \mathrm{~A}-\mathrm{D})$. CAV patients without collaterals displayed significantly increased levels of fibrosis $(9.7 \pm 3.0 \%)$ compared to both controls $(2.9 \pm 1.6 \%, \mathrm{p}<0.001)$ and CAV patients with coronary collaterals $(5.5 \pm 2.7 \%, \mathrm{p}<0.001)$. Patients with CAV and collaterals had reduced interstitial fibrosis compared to those without collaterals $(\mathrm{p}<0.01)$.

Consistent with increased interstitial fibrosis in CAV patients, patients with CAV had higher LVEDP compared to control subjects (Figure 6E). For these analyses LVEDP was measured at the time of CAV diagnosis. CAV patients without collaterals $(27.3 \pm 6.9 \mathrm{mmHg})$ and CAV patients with collaterals $(18.6 \pm 6.3 \mathrm{mmHg}$ ) demonstrated increased LVEDP compared to control subjects $(12.7 \pm 3.6 \mathrm{mmHg}, \mathrm{p}<0.001)$. In addition, $\mathrm{CAV}$ patients without collaterals had significant higher LVEDP compared to CAV patients with collaterals $(p<0.001)$. Linear regression analysis revealed that myocardial fibrosis and microvascular density were significant contributors to LVEDP (Figure 6F-G). Myocardial fibrosis was positively correlated with LVEDP $\left(\mathrm{r}^{2}=0.48, \mathrm{p}<0.001\right)$, and microvascular density was inversely correlated with LVEDP $\left(\mathrm{r}^{2}=0.49, \mathrm{p}<0.001\right)$.

\section{Discussion}

The major finding of this study is that the development of collateral coronary vasculature predicts improved survival and allograft function in patients with moderate or severe CAV. The presence of coronary collaterals correlated with less severe heart failure symptoms as assessed by NYHA classification. Furthermore, coronary collaterals were associated with preserved microvascular density, reduced myocardial fibrosis, and decreased LVEDP.

The prognostic importance of collateral blood vessel development in coronary artery disease and ischemic cardiomyopathy has been well established. Patients who form angiographically visible collateral vasculature have reduced rates of cardiac mortality and major adverse cardiac events, compared to patients without collaterals. In addition, analyses of 
physiological assessment of collateral perfusion as measure by collateral flow index (CFI) have revealed improved outcomes in patients with increased collateral flow ${ }^{23-27}$.

To date, there have been conflicting reports describing whether patients with CAV can develop collateral vasculature. In an angiographic study of $81 \mathrm{CAV}$ patients, Gao et al reported that in the setting of total vessel occlusion only $8 \%$ of patients had angiographically visible coronary collaterals ${ }^{34}$. In contrast to these findings, Bajaj et al described a series of 73 patients with CAV and demonstrated the presence of angiographically visible collaterals in the majority of subjects. In comparison to patients with native CAD, CAV patients had smaller collateral vessels that were described as having a "myocardial blush pattern" 35 . Physiological evaluation using coronary artery occlusion pressure suggested that patients with CAV have identical collateral flow to those with native $\mathrm{CAD}^{36}$. Similarly, a recent study using propensity matching showed that patients with CAV have a collateral flow index that approximates those with native $\mathrm{CAD}^{37}$.

Consistent with the latter observations, we found that the $34 / 60$ (57\%) of patients with CAV had angiographically visible collaterals. The majority of patients had smaller sized collaterals that formed a web like pattern (Figure 1C) consistent with prior reports ${ }^{35}$. Our analysis not only confirmed these prior findings, but also, revealed that the formation of coronary collaterals portends a favorable prognosis in patients with CAV. We further demonstrated that collateral formation served as a powerful independent predictor of survival and cardiovascular outcomes in this patient population.

\section{Importance of the microvasculature}

CAV has been demonstrated to affect the entire length of the coronary blood vessel from the epicardial artery to smaller intramyocardial arterioles. Abnormalities of the epicardial vasculature are readily identifiable by coronary angiography and intravascular ultrasound. Decreased microvascular perfusion has been demonstrated in patients with CAV using TIMI myocardial perfusion grade (TMPG), coronary flow reserve, and PET imaging. These studies additionally show an inverse correlation between epicardial plaque progression and microvasculature dysfunction, suggesting diffuse involvement of the coronary vascular tree ${ }^{38,38,39}$. The importance of microvascular perfusion is supported by the correlation between survival and TIMI myocardial perfusion grade ${ }^{40}$.

In support of the diffuse nature of $\mathrm{CAV}$, analyses of endomyocardial biopsy specimens have revealed histological evidence of intramyocardial arteriole inflammation and endothelial cell injury ${ }^{39,41}$. We further extend these observations to demonstrate that patients with CAV have significant reductions in microvascular density at the arteriole and capillary level.

Intriguingly, patients with angiographically visible collaterals demonstrated expansion of both the small arteriole and capillary compartments, as compared to patients without collaterals. These data raise the possibility that a subgroup of patients with CAV are capable of activating a pro-angiogenic program resulting in simultaneous development of coronary collateral blood vessels as well as preservation of microvascular density. This broad proangiogenic response is associated with improved outcomes, less severe heart failure symptoms, reduced myocardial fibrosis and lower LVEDP. The mechanistic explanation for which only a portion of patients undergo an expansion of their coronary vasculature in response to $\mathrm{CAV}$ is unknown and will be a focus of future studies.

\section{Study limitations}

The primary limitation of this study is the sample size $(n=59)$ and retrospective study design. We chose to only include patients with moderate to severe CAV. This was done to enrich for 
a patient population that definitively had CAV with a relatively high rate of adverse cardiovascular events at the expense of a smaller sample size. As a result, this approach may overestimate the mortality rate and exaggerate differences in event rates between patients with and without collaterals. Despite the small sample size, we were fortunate to capture a well-balanced group of patients with similar demographics and comborbidities and much of our data were consistent with previous reports. Pravastatin and simvastatin reduce disease progression and mortality in clinical trials ${ }^{12,13}$ and azathioprine, as compared with mycophenolate, has previously been associated with an increased incidence of CAV ${ }^{16,42}$. The relationship between prednisone therapy and outcomes in patients in CAV has not yet been reported and may be explained by direct effects of this agent such as reduced angiogenesis and poor wound healing ${ }^{43}$. Alternatively, ongoing use of prednisone may serve as a surrogate marker of chronic rejection and more aggressive transplant vasculopathy.

The use of coronary angiography to identify patients with coronary collaterals represents a potential limitation of this study. Angiographic identification of collateral blood vessels is a less robust method compared to invasive measurements of collateral perfusion ${ }^{26}$. Since coronary flow measurements were not available for the majority of patients, coronary angiography was utilized to identify patients with collaterals.

An additional limitation of this study involves the use of RV endomyocardial biopsy specimens. Our findings are subject to potential sampling error. That is, we are making the assumption that results obtained from RV endomyocardial biopsy specimens are reflective of changes throughout the heart including the LV. In contrast, to native ischemic heart disease, the impact of potential sampling error may be minimized as CAV tends to globally affect the myocardium ${ }^{6}$.

\section{Implications for prognosis and treatment}

In terms of prognostic data, disease progression as assessed by intravascular ultrasound appears to provide the most definitive information and is associated with the development of allograft failure and mortality ${ }^{44,45}$. Additionally, smaller clinical studies revealed that measurement of myocardial perfusion by either PET or TMPG is associated with both plaque volume and clinical outcomes 39,40 . These studies implicate the rate of plaque growth and absolute myocardial perfusion as central components of disease progression, each contributing to overall prognosis.

In this study, we add to the available prognostic data for the CAV patient population and show that coronary collateralization as assessed by angiography is associated with significantly improved outcomes including heart failure symptoms and mortality. The presence of coronary collateral vasculature was the single strongest prognostic predictor of survival in our cohort and was independently associated with clinical outcomes. We further demonstrated that patients who form coronary collateral blood vessels are more likely to have preserved microvascular density.

Together the above data, suggest that the intrinsic ability to grow new coronary vasculature on the large and small vessel level is central to outcomes in patients with CAV. Whether this is reflected by the previous findings of improved prognosis in patients with increase myocardial perfusion remains to be rigorously studied. It is intriguing to speculate that quantification of myocardial perfusion as assayed by modalities such as cardiac PET and/or MRI may help to better predict outcomes in patients with CAV. Such an approach has the potential to identify patients who may benefit from retransplantation from those who would do well with medical therapy. 
These findings have additional implications with respect to novel therapies for patients with CAV. Therapeutic angiogenesis has been proposed as a biological approach for revascularization in patients with ischemic cardiomyopathy and peripheral vascular disease. This strategy involves administration of growth factors or gene therapy to promote angiogenesis and collateral growth. Despite initial promise in phase I and II studies, larger clinical trials utilizing growth factors such as fibroblast growth factors (FGF2 and FGF4), vascular endothelial growth factors (VEGF-A and VEGF-B), and colony stimulating growth factors (G-CSF and GM-CSF) have largely been disappointing 46,47 . Whether these proangiogenic factors encompassed the most ideal targets remain a topic of much debate.

It is intriguing to consider the possibility that elucidation of the molecular mechanism by which coronary collaterals grow and mature in patients with CAV, may provide critical insights into how these blood vessels form in patients with ischemic heart disease. The identification of molecules that are sufficient to promote coronary collateral growth may lead to the development of novel therapies for both of these diseases. It is conceivable that pro-angiogenic therapy may promote both the development of collateral blood vessels in patients who are not able to form collateral vasculature as well as enhance collateral growth and maturation in those who have intrinsic collaterals.

\section{Acknowledgments}

\section{Funding sources.}

This project was made possible by the Washington University Institute of Clinical and Translational Sciences grant UL1 RR024992 from the National Center for Research Resources (NCRR)/National Center for Advancing Translational Sciences (NCATS), a component of the National Institutes of Health (NIH), as well as funding from the NIH (HL111094). KL was supported by NIH T32 HL007081 and the Physician Scientist Training Program.

\section{Reference List}

1. Taylor DO, Stehlik J, Edwards LB, Aurora P, Christie JD, Dobbels F, Kirk R, Kucheryavaya AY, Rahmel AO, Hertz MI. Registry of the International Society for Heart and Lung Transplantation: Twenty-sixth Official Adult Heart Transplant Report-2009. J Heart Lung Transplant. 2009; 28:1007-1022. [PubMed: 19782283]

2. Colvin-Adams M, Agnihotri A. Cardiac allograft vasculopathy: current knowledge and future direction. Clin Transplant. 2011; 25:175-184. [PubMed: 21457328]

3. Kass M, Haddad H. Cardiac allograft vasculopathy: pathology, prevention and treatment. Curr Opin Cardiol. 2006; 21:132-137. [PubMed: 16470150]

4. Schmauss D, Weis M. Cardiac allograft vasculopathy: recent developments. Circulation. 2008; 117:2131-2141. [PubMed: 18427143]

5. Billingham ME. Histopathology of graft coronary disease. J Heart Lung Transplant. 1992; 11:S38S44. [PubMed: 1622997]

6. Rahmani M, Cruz RP, Granville DJ, McManus BM. Allograft vasculopathy versus atherosclerosis. Circ Res. 2006; 99:801-815. [PubMed: 17038650]

7. Tuzcu EM, De Franco AC, Goormastic M, Hobbs RE, Rincon G, Bott-Silverman C, McCarthy P, Stewart R, Mayer E, Nissen SE. Dichotomous pattern of coronary atherosclerosis 1 to 9 years after transplantation: insights from systematic intravascular ultrasound imaging. J Am Coll Cardiol. 1996; 27:839-846. [PubMed: 8613612]

8. Kapadia SR, Ziada KM, L'Allier PL, Crowe TD, Rincon G, Hobbs RE, Bott-Silverman C, Young JB, Nissen SE, Tuzcu EM. Intravascular ultrasound imaging after cardiac transplantation: advantage of multi-vessel imaging. J Heart Lung Transplant. 2000; 19:167-172. [PubMed: 10703693]

9. St Goar FG, Pinto FJ, Alderman EL, Valantine HA, Schroeder JS, Gao SZ, Stinson EB, Popp RL. Intracoronary ultrasound in cardiac transplant recipients. In vivo evidence of "angiographically silent" intimal thickening. Circulation. 1992; 85:979-987. [PubMed: 1537134] 
10. Mehra MR, Ventura HO, Stapleton DD, Smart FW, Collins TC, Ramee SR. Presence of severe intimal thickening by intravascular ultrasonography predicts cardiac events in cardiac allograft vasculopathy. J Heart Lung Transplant. 1995; 14:632-639. [PubMed: 7578168]

11. Spes CH, Mudra H, Schnaack SD, Klauss V, Reichle FM, Uberfuhr P, Theisen K, Angermann CE. Dobutamine stress echocardiography for noninvasive diagnosis of cardiac allograft vasculopathy: a comparison with angiography and intravascular ultrasound. Am J Cardiol. 1996; 78:168-174. [PubMed: 8712138]

12. Kobashigawa JA, Katznelson S, Laks H, Johnson JA, Yeatman L, Wang XM, Chia D, Terasaki PI, Sabad A, Cogert GA. Effect of pravastatin on outcomes after cardiac transplantation. N Engl J Med. 1995; 333:621-627. [PubMed: 7637722]

13. Mehra MR, Uber PA, Vivekananthan K, Solis S, Scott RL, Park MH, Milani RV, Lavie CJ. Comparative beneficial effects of simvastatin and pravastatin on cardiac allograft rejection and survival. J Am Coll Cardiol. 2002; 40:1609-1614. [PubMed: 12427413]

14. Mehra MR, Ventura HO, Smart FW, Collins TJ, Ramee SR, Stapleton DD. An intravascular ultrasound study of the influence of angiotensin-converting enzyme inhibitors and calcium entry blockers on the development of cardiac allograft vasculopathy. Am J Cardiol. 1995; 75:853-854. [PubMed: 7717300]

15. Schroeder JS, Gao SZ, Alderman EL, Hunt SA, Johnstone I, Boothroyd DB, Wiederhold V, Stinson EB. A preliminary study of diltiazem in the prevention of coronary artery disease in hearttransplant recipients. N Engl J Med. 1993; 328:164-170. [PubMed: 8417382]

16. Kaczmarek I, Ertl B, Schmauss D, Sadoni S, Knez A, Daebritz S, Meiser B, Reichart B. Preventing cardiac allograft vasculopathy: long-term beneficial effects of mycophenolate mofetil. J Heart Lung Transplant. 2006; 25:550-556. [PubMed: 16678034]

17. Keogh A, Richardson M, Ruygrok P, Spratt P, Galbraith A, O'Driscoll G, Macdonald P, Esmore D, Muller D, Faddy S. Sirolimus in de novo heart transplant recipients reduces acute rejection and prevents coronary artery disease at 2 years: a randomized clinical trial. Circulation. 2004; 110:2694-2700. [PubMed: 15262845]

18. Eisen HJ, Tuzcu EM, Dorent R, Kobashigawa J, Mancini D, Valantine-von Kaeppler HA, Starling RC, Sorensen K, Hummel M, Lind JM, Abeywickrama KH, Bernhardt P. Everolimus for the prevention of allograft rejection and vasculopathy in cardiac-transplant recipients. N Engl J Med. 2003; 349:847-858. [PubMed: 12944570]

19. Potena L, Holweg CT, Chin C, Luikart H, Weisshaar D, Narasimhan B, Fearon WF, Lewis DB, Cooke JP, Mocarski ES, Valantine HA. Acute rejection and cardiac allograft vascular disease is reduced by suppression of subclinical cytomegalovirus infection. Transplantation. 2006; 82:398405. [PubMed: 16906040]

20. Halle AA III, DiSciascio G, Massin EK, Wilson RF, Johnson MR, Sullivan HJ, Bourge RC, Kleiman NS, Miller LW, Aversano TR. Coronary angioplasty, atherectomy and bypass surgery in cardiac transplant recipients. J Am Coll Cardiol. 1995; 26:120-128. [PubMed: 7797740]

21. Bader FM, Kfoury AG, Gilbert EM, Barry WH, Humayun N, Hagan ME, Thomas H, Renlund D. Percutaneous coronary interventions with stents in cardiac transplant recipients. J Heart Lung Transplant. 2006; 25:298-301. [PubMed: 16507423]

22. Segovia J, Gomez-Bueno M, Alonso-Pulpon L. Treatment of allograft vasculopathy in heart transplantation. Expert Opin Pharmacother. 2006; 7:2369-2383. [PubMed: 17109612]

23. Billinger M. Physiologically assessed coronary collateral flow and adverse cardiac ischemic events: a follow-up study in 403 patients with coronary artery disease. 2002

24. Nathoe HM. Determinants and prognostic significance of collaterals in patients undergoing coronary revascularization. 2006

25. Steg PG. Impact of collateral flow to the occluded infarct-related artery on clinical outcomes in patients with recent myocardial infarction: a report from the randomized occluded artery trial. 2010

26. Meier P, Gloekler S, Zbinden R, Beckh S, de Marchi SF, Zbinden S, Wustmann K, Billinger M, Vogel R, Cook S, Wenaweser P, Togni M, Windecker S, Meier B, Seiler C. Beneficial effect of recruitable collaterals: a 10 -year follow-up study in patients with stable coronary artery disease 
undergoing quantitative collateral measurements. Circulation. 2007; 116:975-983. [PubMed: 17679611]

27. Meier P, Hemingway H, Lansky AJ, Knapp G, Pitt B, Seiler C. The impact of the coronary collateral circulation on mortality: a meta-analysis. Eur Heart J. 2012; 33:614-621. [PubMed: 21969521]

28. Mehra MR. International Society for Heart and Lung Transplantation working formulation of a standardized nomenclature for cardiac allograft vasculopathy-2010. 2010

29. Concato J, Peduzzi P, Holford TR, Feinstein AR. Importance of events per independent variable in proportional hazards analysis. I. Background, goals, and general strategy. J Clin Epidemiol. 1995; 48:1495-1501. [PubMed: 8543963]

30. Peduzzi P, Concato J, Feinstein AR, Holford TR. Importance of events per independent variable in proportional hazards regression analysis. II. Accuracy and precision of regression estimates. J Clin Epidemiol. 1995; 48:1503-1510. [PubMed: 8543964]

31. Peduzzi P, Concato J, Kemper E, Holford TR, Feinstein AR. A simulation study of the number of events per variable in logistic regression analysis. J Clin Epidemiol. 1996; 49:1373-1379. [PubMed: 8970487]

32. Milano AD, Faggian G, Dodonov M, Golia G, Tomezzoli A, Bortolotti U, Mazzucco A. Prognostic value of myocardial fibrosis in patients with severe aortic valve stenosis. J Thorac Cardiovasc Surg. 2012

33. Zannad F, Rossignol P, Iraqi W. Extracellular matrix fibrotic markers in heart failure. Heart Fail Rev. 2010; 15:319-329. [PubMed: 19404737]

34. Gao SZ, Alderman EL, Schroeder JS, Silverman JF, Hunt SA. Accelerated coronary vascular disease in the heart transplant patient: coronary arteriographic findings. J Am Coll Cardiol. 1988; 12:334-340. [PubMed: 3292629]

35. Bajaj S, Shah A, Crandall C, Ibrahim H, Vetrovec G, DiSciascio G, Guerraty A, Salter DR, Mohanty PK. Coronary collateral circulation in the transplanted heart. Circulation. 1993; 88:II263-II269. [PubMed: 8222164]

36. Johnson TH, Das GS, McGinn AL, Christensen BV, Meyer SM, Wilson RF. Physiologic assessment of the coronary collateral circulation in transplanted human hearts. J Heart Lung Transplant. 1994; 13:840-846. [PubMed: 7803425]

37. Rutz T, Gloekler S, de Marchi SF, Traupe T, Meier P, Eshtehardi P, Cook S, Vogel R, Mohacsi P, Seiler C. Coronary collateral function in the transplanted heart: propensity score matching with coronary artery disease. Heart. 2011; 97:557-563. [PubMed: 21270076]

38. Lee CM, Wu YW, Jui HY, Yen RF, Tzen KY, Chou NK, Wang SS. Intravascular ultrasound correlates with coronary flow reserve and predicts the survival in angiographically normal cardiac transplant recipients. Cardiology. 2008; 109:93-98. [PubMed: 17664873]

39. Wu YW, Chen YH, Wang SS, Jui HY, Yen RF, Tzen KY, Chen MF, Lee CM. PET assessment of myocardial perfusion reserve inversely correlates with intravascular ultrasound findings in angiographically normal cardiac transplant recipients. J Nucl Med. 2010; 51:906-912. [PubMed: 20484427]

40. Potluri SP, Mehra MR, Uber PA, Park MH, Scott RL, Ventura HO. Relationship among epicardial coronary disease, tissue myocardial perfusion, and survival in heart transplantation. J Heart Lung Transplant. 2005; 24:1019-1025. [PubMed: 16102436]

41. Clausell N, Butany J, Molossi S, Lonn E, Gladstone P, Rabinovitch M, Daly PA. Abnormalities in intramyocardial arteries detected in cardiac transplant biopsy specimens and lack of correlation with abnormal intracoronary ultrasound or endothelial dysfunction in large epicardial coronary arteries. J Am Coll Cardiol. 1995; 26:110-119. [PubMed: 7541058]

42. Dandel M, Jasaityte R, Lehmkuhl H, Knosalla C, Hetzer R. Maintenance immunosuppression with mycophenolate mofetil: long-term efficacy and safety after heart transplantation. Transplant Proc. 2009; 41:2585-2588. [PubMed: 19715979]

43. Schacke H, Docke WD, Asadullah K. Mechanisms involved in the side effects of glucocorticoids. Pharmacol Ther. 2002; 96:23-43. [PubMed: 12441176]

44. Tuzcu EM, Kapadia SR, Sachar R, Ziada KM, Crowe TD, Feng J, Magyar WA, Hobbs RE, Starling RC, Young JB, McCarthy P, Nissen SE. Intravascular ultrasound evidence of 
angiographically silent progression in coronary atherosclerosis predicts long-term morbidity and mortality after cardiac transplantation. J Am Coll Cardiol. 2005; 45:1538-1542. [PubMed: 15862431]

45. Kobashigawa JA, Tobis JM, Starling RC, Tuzcu EM, Smith AL, Valantine HA, Yeung AC, Mehra MR, Anzai H, Oeser BT, Abeywickrama KH, Murphy J, Cretin N. Multicenter intravascular ultrasound validation study among heart transplant recipients: outcomes after five years. J Am Coll Cardiol. 2005; 45:1532-1537. [PubMed: 15862430]

46. Grundmann S, Piek JJ, Pasterkamp G, Hoefer IE. Arteriogenesis: basic mechanisms and therapeutic stimulation. Eur J Clin Invest. 2007; 37:755-766. [PubMed: 17764463]

47. Seiler C. The human coronary collateral circulation. Eur J Clin Invest. 2010; 40:465-476. [PubMed: 20534067] 


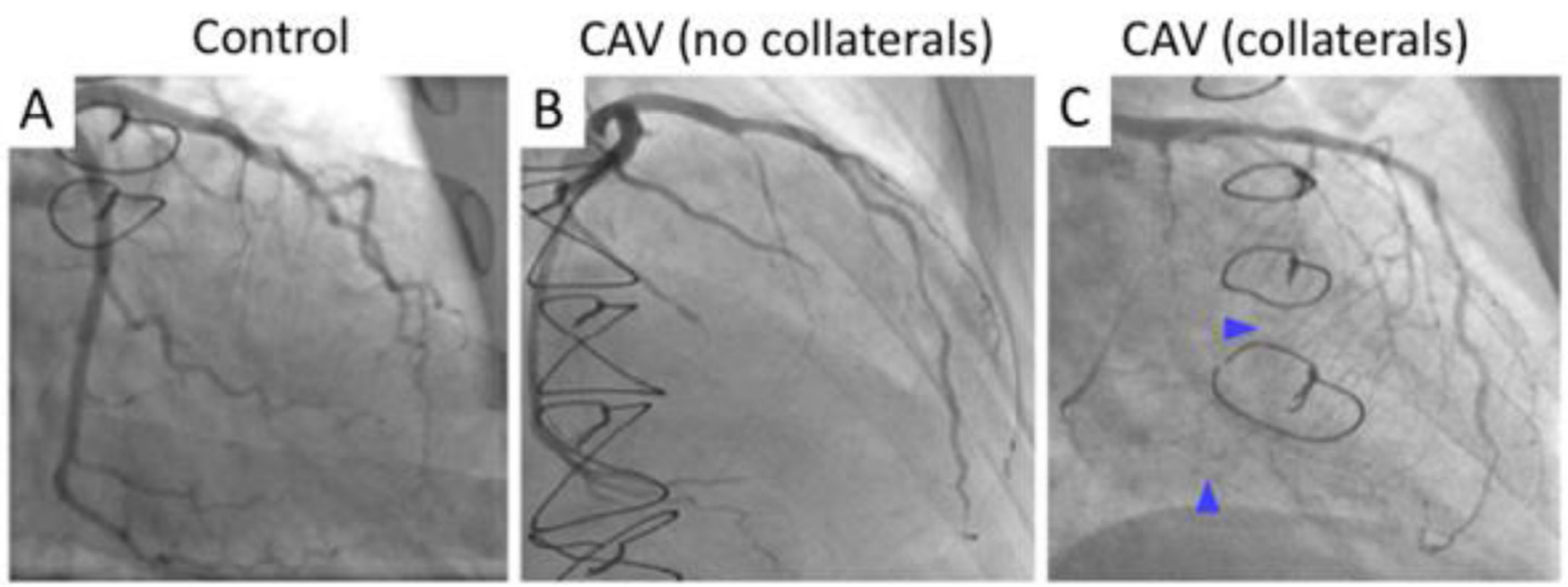

D

Transplanted at BJH 1994-2008 $n=330$

Moderate or Severe CAV $n=63$<smiles>[AlH2]</smiles>

Available

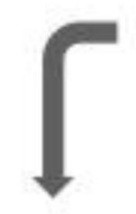

No collaterals

$$
n=25
$$

Transplanted elsewhere 1994-2008 $n=155$

No angiographic data

$$
n=4
$$

Figure 1. Study design

A-C, Representative coronary angiograms from patients with (A) normal coronary arteries, (B) coronary allograft vasculopathy without collaterals, (C) and coronary allograft vasculopathy with collaterals (C). D, Schematic describing the number of patients that were screened, included in this study, and assigned to either the collateral or no collateral group. 

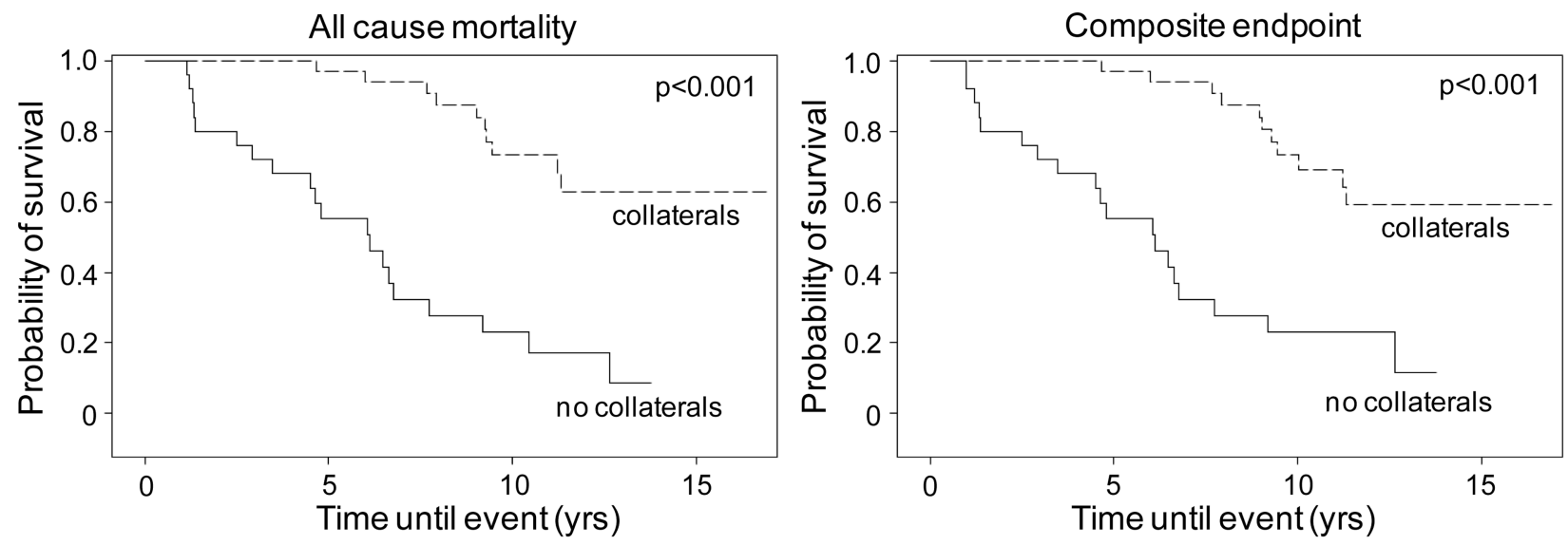

\begin{tabular}{|lrrrrrrrrr|}
\hline collaterals & 34 & 32 & 18 & 7 & 34 & 32 & 18 & 7 \\
no collaterals & 25 & 13 & 5 & 0 & 25 & 13 & 5 & 0 \\
\hline
\end{tabular}

Figure 2. Coronary collaterals predict improved outcomes in patients with CAV

Kaplan-Meier survival curves demonstrated reduced rates of all cause mortality (left) and the composite endpoint of all cause mortality, retransplantation or chronic inotrope use (right). The dashed line indicates the collateral group and the solid line indicates the no collateral group. 

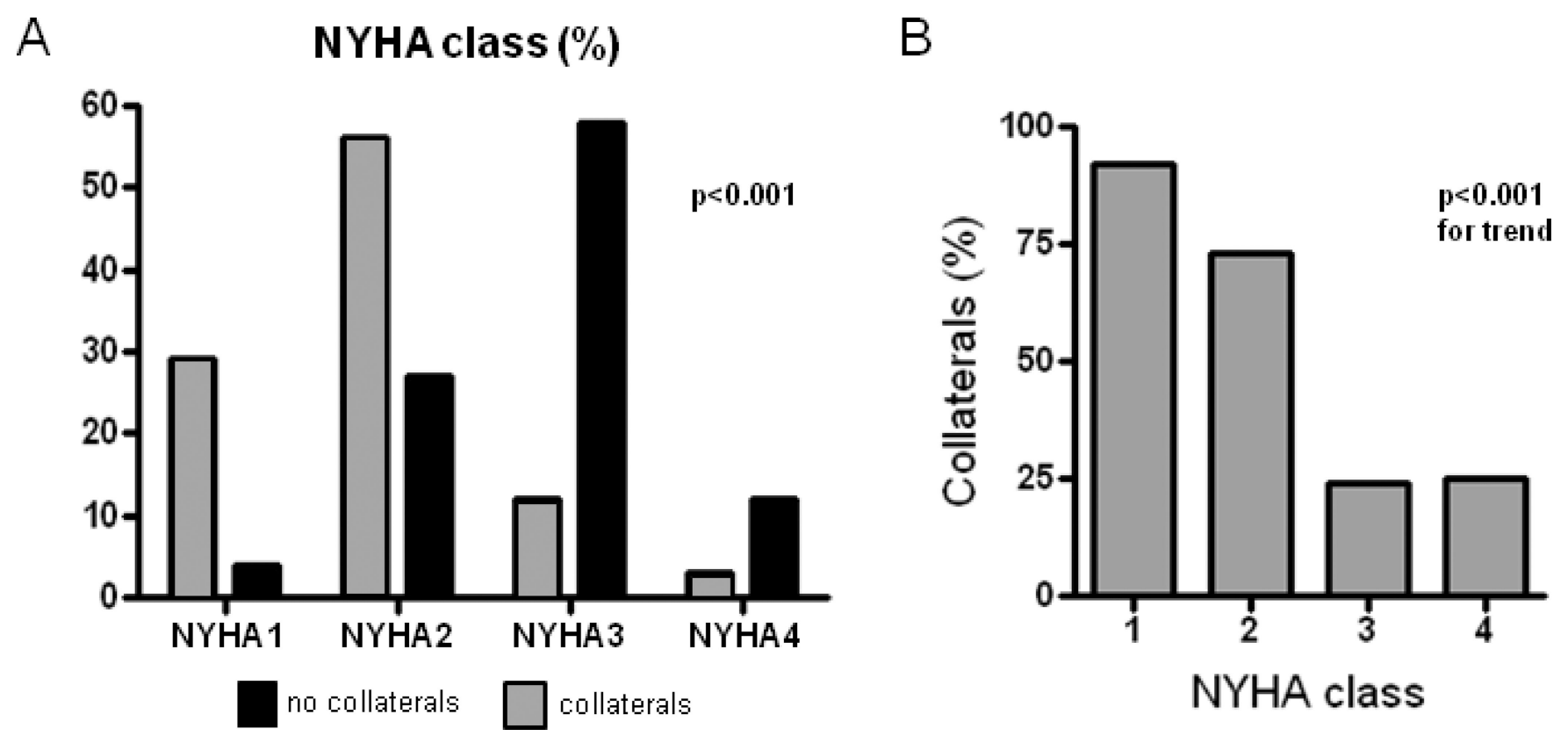

Figure 3. Reduced heart failure symptoms in patients with coronary collaterals

A, Heart failure symptoms as defined by NYHA class. Patients without coronary collaterals (black bars) had predominately NYHA class III symptoms while patients with coronary collaterals (gray bars) had predominately NYHA class II symptoms. B, Trend analysis demonstrating a statistically significant relationship between collateral formation and NYHA class. 
A

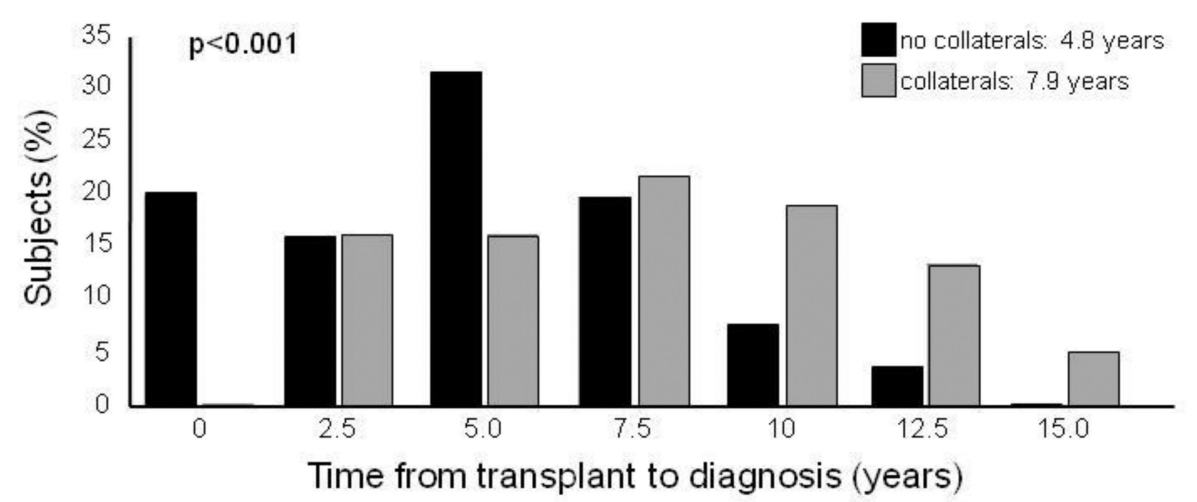

B
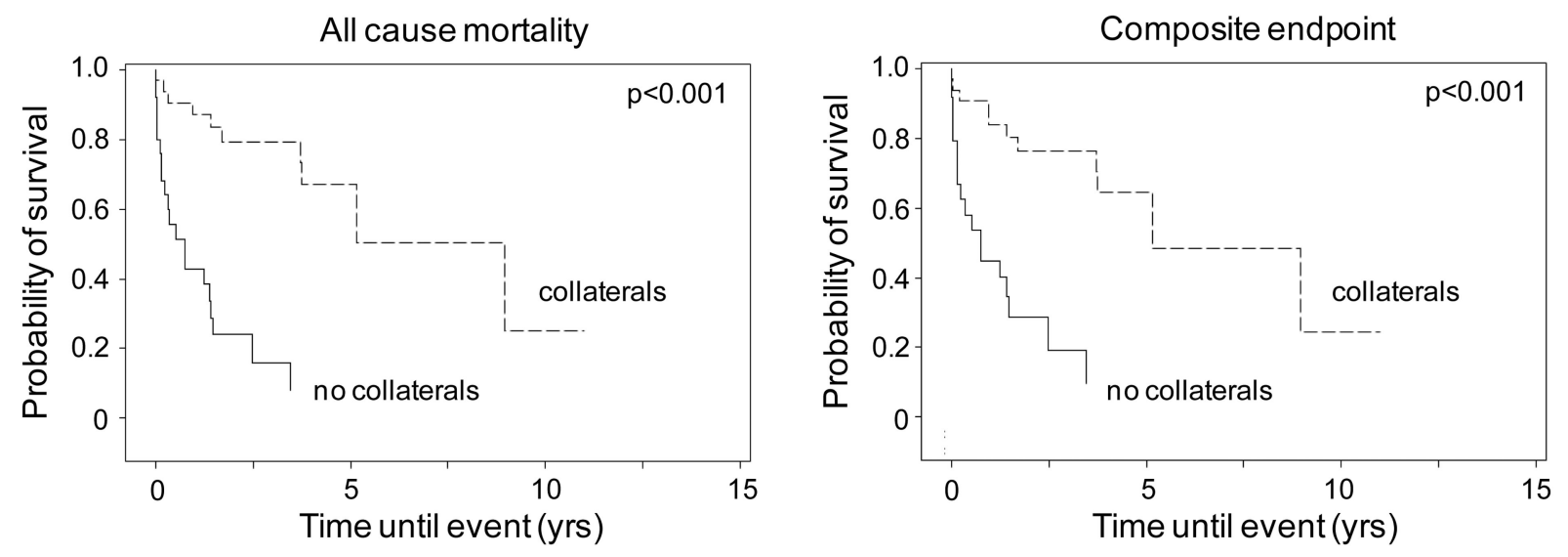

\begin{tabular}{|lccccccccc}
\hline collaterals & 34 & 5 & 1 & 0 & 34 & 0 & 1 \\
no collaterals & 25 & 0 & 0 & 0 & 25 & 0
\end{tabular}

All cause mortality

Collaterals
Time to diagnosis
Interaction term

Interaction term

\begin{tabular}{crr} 
HR & 95\% Cl & p-value \\
0.09 & $0.03-0.25$ & $<0.001$ \\
0.27 & $0.14-0.50$ & $<0.001$ \\
1.10 & $1.03-1.18$ & 0.007 \\
\hline
\end{tabular}

Figure 4. Interaction between coronary collateral formation and timing of CAV diagnosis

A Histogram depicting the distribution of when the diagnosis of CAV was established in patients without collaterals (black bars) and with collaterals (gray bars). Patients with coronary collaterals were diagnosed later than those without collaterals. Time until event indicates the number of years from transplant to CAV diagnosis. B, Kaplan-Meier survival curves demonstrating that compared to patients without collaterals (solid line), patients with coronary collaterals (dashed lines) have reduced rates of all cause mortality (left) and the composite endpoint (right) after the diagnosis of CAV was made. Time until event denotes the time from CAV diagnosis to each endpoint. $\mathbf{C}$, Cox multivariable analysis revealing that 
the presence of coronary collaterals predicted reduced incidence of the all cause mortality and the composite endpoint in patients with CAV independent of the time to diagnosis. There was a significant interaction between time to event and the time to diagnosis for both endpoints. 


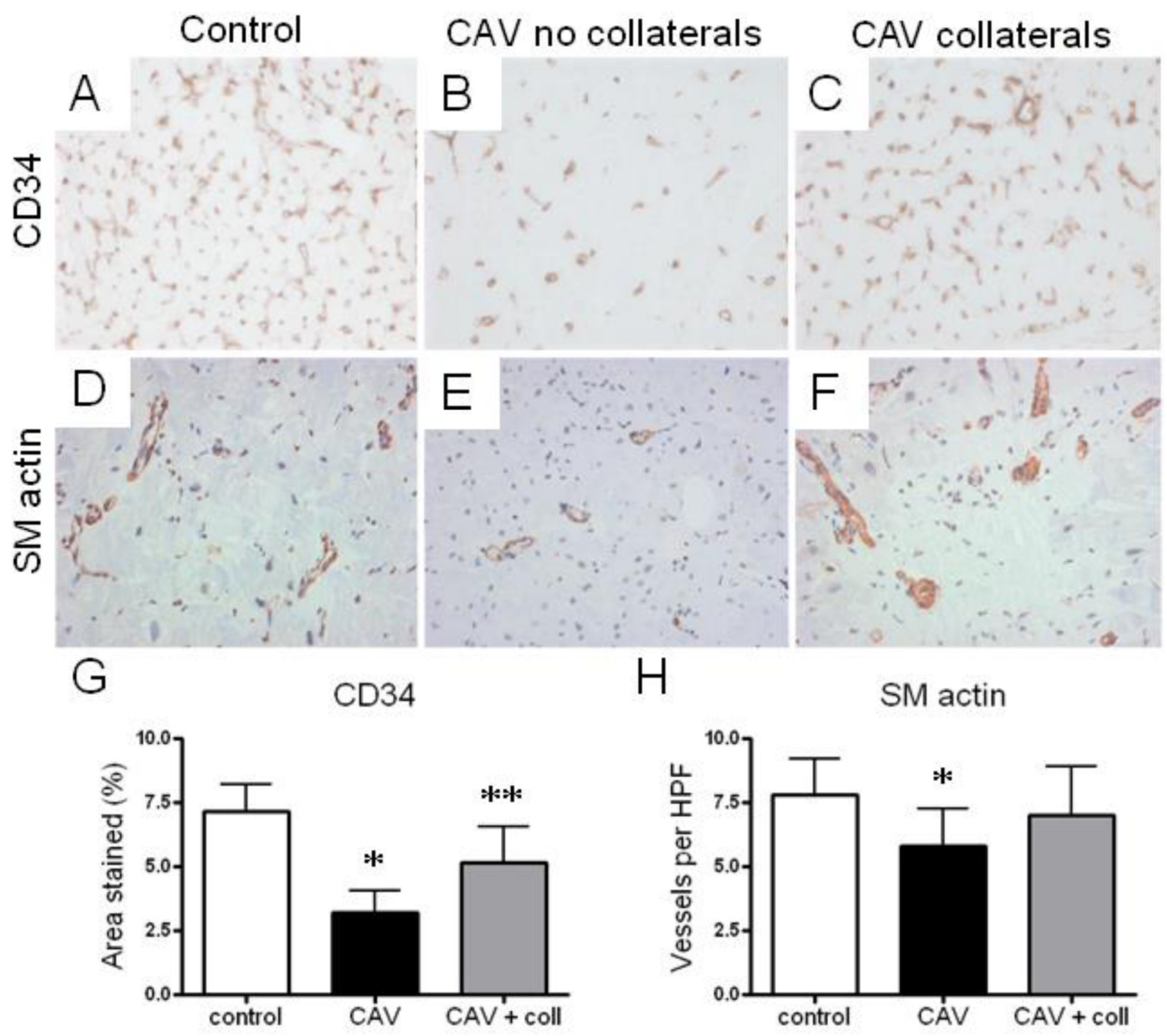

Figure 5. Alterations in microvascular density in patients with $\mathrm{CAV}$ according to the presence of collaterals

A-C, Compared to controls (A), patients with CAV (B) have reduced microvascular density as defined by CD34 immunostaining (brown). Patients with CAV and coronary collaterals

(C) have partially preserved microvascular density compared to those without collaterals

(B). D-F, Smooth muscle (SM) actin immunostaining (brown) demonstrating reduced numbers of arterioles in patients with CAV (E) compared to controls (D). In contrast, patients with CAV and collaterals $(\mathrm{F})$ have no change in arteriolar density compared to controls (D). G-H, Quantitation of microvascular density (G) and SM actin containing blood vessels $(\mathrm{H})$. Microvascular density is expressed as percent area of CD34 staining. SMA containing blood vessels were quantified as the number of blood vessels per 20X field. * denotes $\mathrm{p}<0.01$ compared to controls, $* *$ denotes $\mathrm{p}<0.01$ compared to controls and patients with CAV lacking collaterals. Error bars indicate standard deviations. 


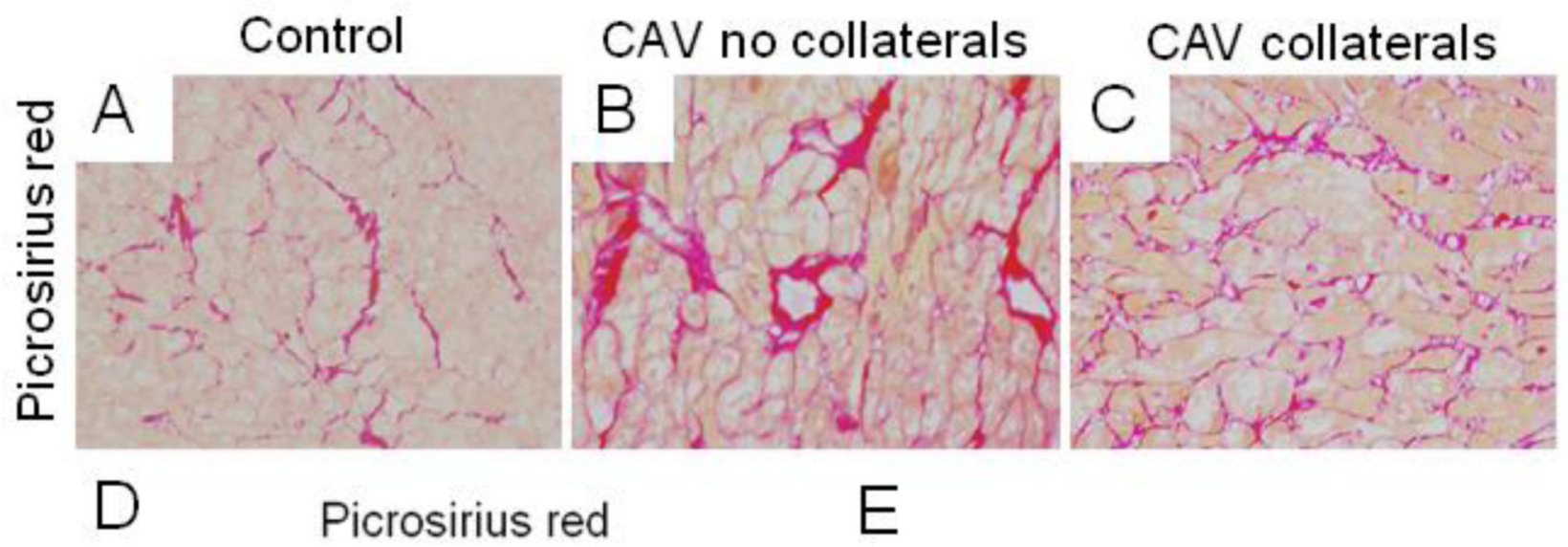

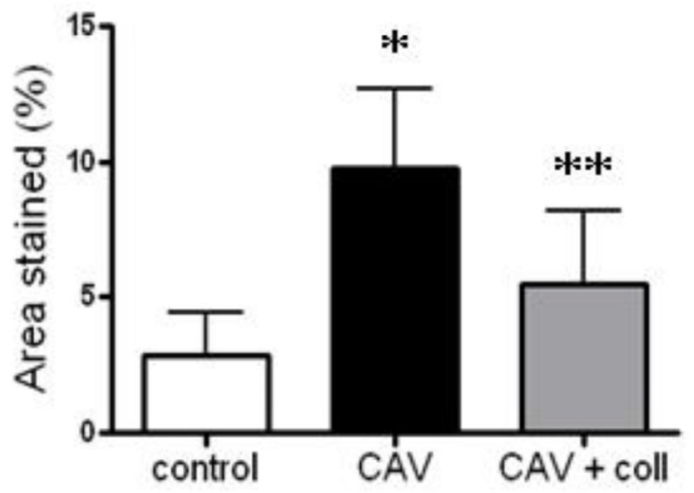

$\mathrm{F}$

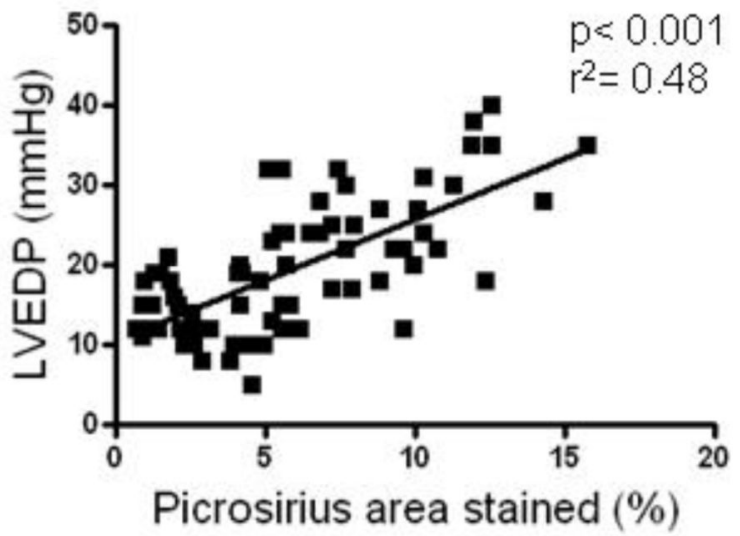

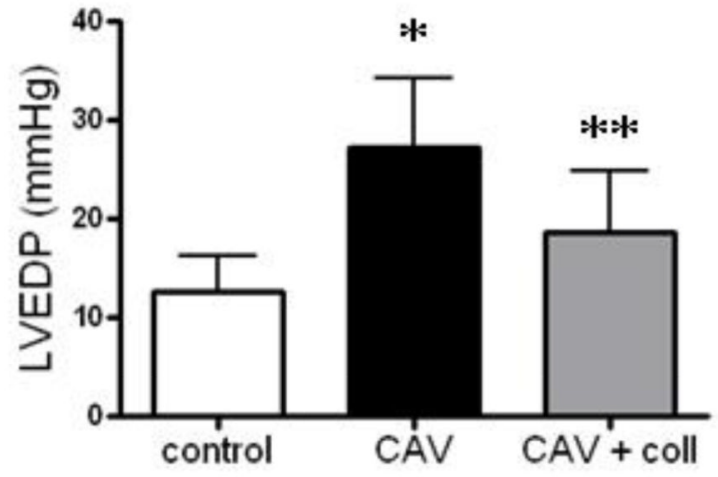

G

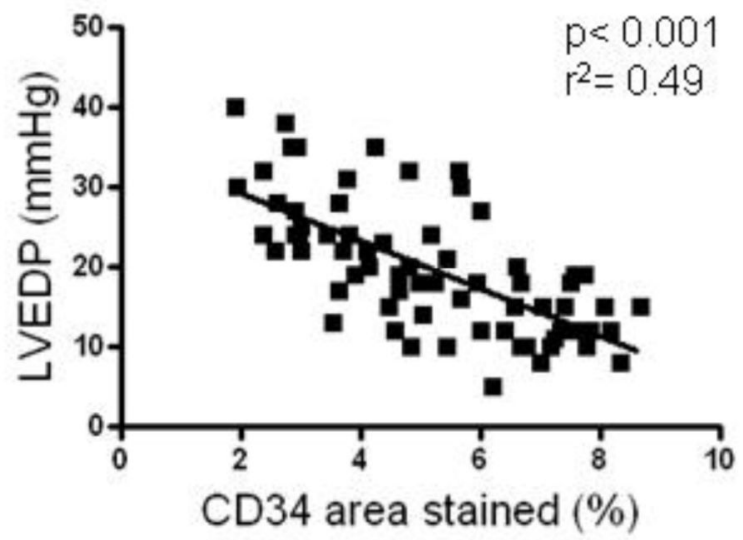

Figure 6. Myocardial fibrosis and LVEDP in CAV patients according to the presence of collaterals

A-C, Picrosirius red staining (red) demonstrating increased interstitial fibrosis in patients with CAV (B) compared to controls (A). Patients with CAV and collaterals (C) have reduced interstitial fibrosis compared to those without collaterals (B). D, Quantitation of interstitial fibrosis expressed as percent area of Picrosirius red staining. E, Increased LVEDP in CAV patients compared to controls. Patients coronary collaterals have reduced LVEDP compared to patients without collaterals. F-G, Linear regression analysis describing the relationship between myocardial fibrosis, microvascular density and LVEDP. * denotes 
$\mathrm{p}<0.01$ compared to controls, and $* *$ denotes $\mathrm{p}<0.01$ compared to controls and patients with CAV lacking collaterals. Error bars indicate standard deviations. 
Table 1

Demographics of patients with CAV in the collateral and no collateral groups.

\begin{tabular}{|c|c|c|c|}
\hline & no collaterals $(\%) n=25$ & collaterals $(\%) n=34$ & p-value \\
\hline Age (yr) & $42.1 \pm 15.2$ & $48.0 \pm 12.0$ & 0.08 \\
\hline Male & $16(64)$ & $24(71)$ & 0.78 \\
\hline Caucasion & $21(84)$ & $24(71)$ & 0.35 \\
\hline NICM & $17(68)$ & $20(59)$ & 0.59 \\
\hline ICM & $8(32)$ & $14(41)$ & 0.59 \\
\hline Diabetes & $12(48)$ & $14(41)$ & 0.79 \\
\hline ESRD & $4(16)$ & $8(24)$ & 0.53 \\
\hline Time to Dx (yr) & $4.8 \pm 3.1$ & $7.9 \pm 3.6$ & 0.001 \\
\hline CMV & $14(56)$ & $20(59)$ & 1.00 \\
\hline Rejection & $8(33)$ & $6(18)$ & 0.22 \\
\hline Blood type & & & 0.27 \\
\hline $\mathbf{A}$ & $10(42)$ & $19(59)$ & \\
\hline B & $3(13)$ & $6(19)$ & \\
\hline $\mathbf{A B}$ & $3(13)$ & $1(3)$ & \\
\hline $\mathbf{O}$ & $9(33)$ & $6(19)$ & \\
\hline Tacrolimus & $17(71)$ & $22(65)$ & 0.78 \\
\hline Cyclosporine & $5(21)$ & $11(32)$ & 0.39 \\
\hline Azathioprine & $6(25)$ & $6(18)$ & 0.53 \\
\hline Mycophenolate & $11(46)$ & $13(38)$ & 0.60 \\
\hline Prednisone & $13(54)$ & $18(53)$ & 1.00 \\
\hline Valganciclovir & $4(17)$ & $3(9)$ & 0.43 \\
\hline Sirolimus & $7(29)$ & $10(29)$ & 1.00 \\
\hline Statin & $18(75)$ & $29(85)$ & 0.50 \\
\hline ACEi/ARB & $10(42)$ & $19(56)$ & 0.42 \\
\hline$\beta$-blocker & $10(42)$ & $16(47)$ & 0.79 \\
\hline Spironolactone & $2(8)$ & $4(12)$ & 1.00 \\
\hline Heart rate & $104 \pm 16.6$ & $91.1 \pm 15.4$ & 0.003 \\
\hline Systolic BP & $120 \pm 17.0$ & $123 \pm 18.9$ & 0.76 \\
\hline Diastolic BP & $84.8 \pm 9.8$ & $78.8 \pm 9.7$ & 0.03 \\
\hline EF $(\%)$ & $44.7 \pm 12.0$ & $49.7 \pm 12.5$ & 0.10 \\
\hline PCI & $7(28)$ & $14(41)$ & 0.41 \\
\hline
\end{tabular}




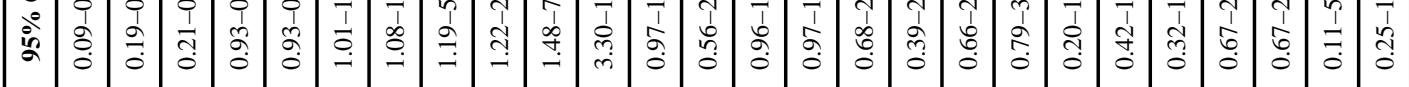

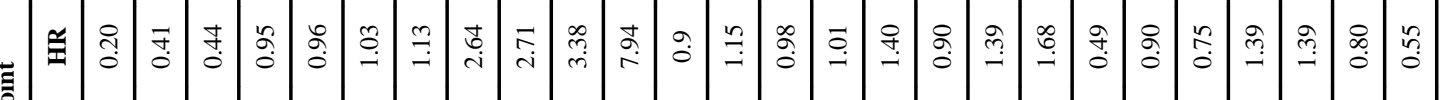

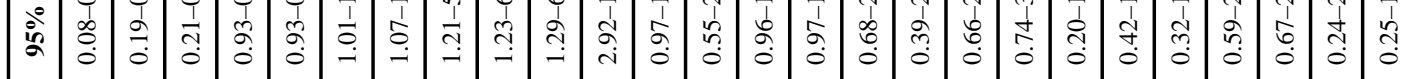
I) 尝

. 

a

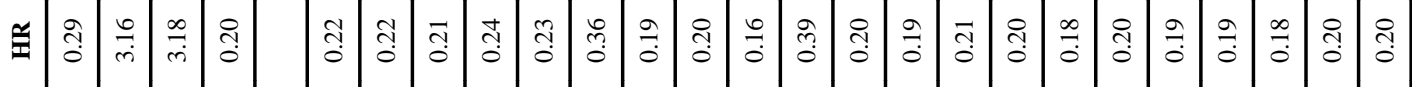

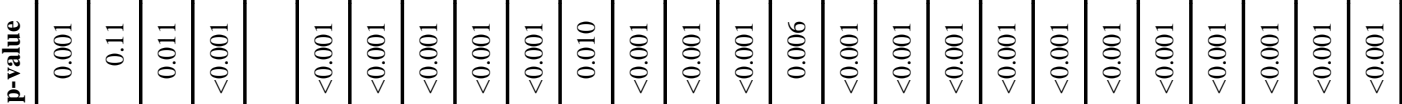

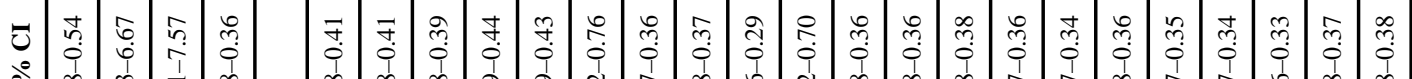

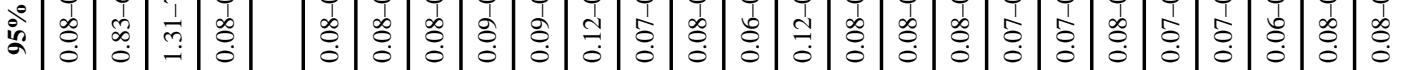

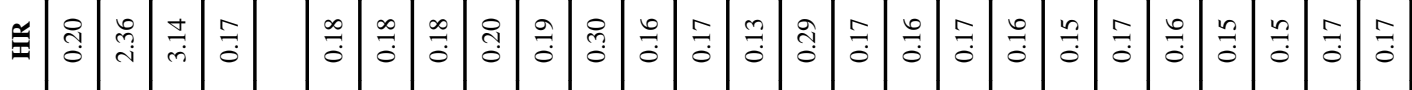
旅

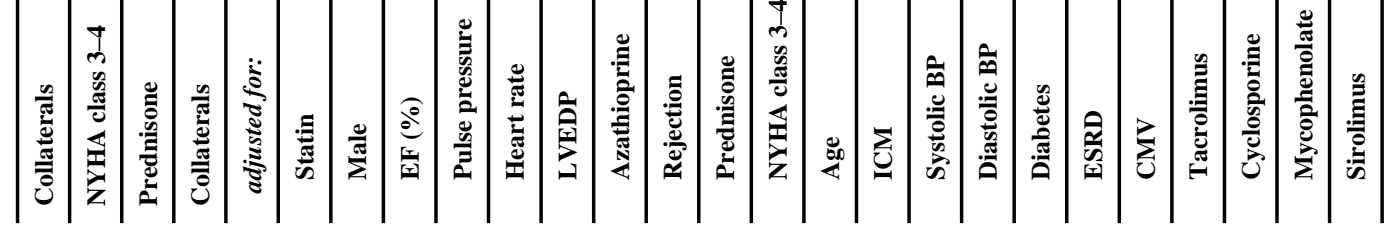




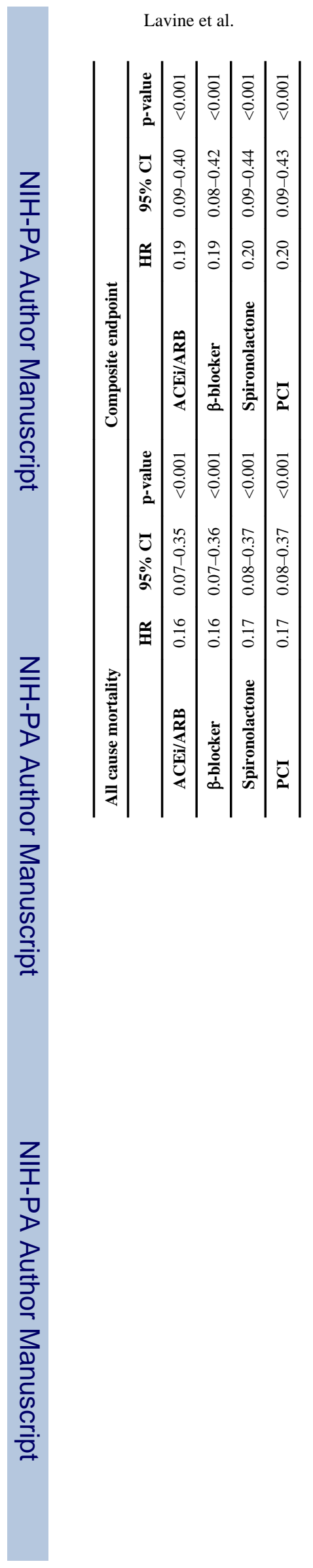

Circ Heart Fail. Author manuscript; available in PMC 2013 December 14. 